\title{
On a singular time-fractional order wave equation with Bessel operator and Caputo derivative
}

\author{
Said Mesloub*, Imed Bachar \\ King Saud University, College of Science, Mathematics Department, P. O. Box 2455, Riyadh 11451, Saudi Arabia. \\ Communicated by B. Samet
}

\begin{abstract}
This paper deals with the study of the well-posedness of a mixed fractional problem for the wave equation defined in a bounded space domain. The fractional time derivative is described in the Caputo sense. We prove the existence and uniqueness of solution as well as its dependence on the given data. Our results develop and show the efficiency and effectiveness of the functional analysis method when we deal with fractional partial differential equations instead of the nonfractional equations which have been extensively studied by many authors during the last three decades. (C)2017 All rights reserved.
\end{abstract}

Keywords: Caputo derivative, solvability of the problem, fractional differential equation, initial boundary value problem. 2010 MSC: 35D35, 35L20.

\section{Introduction}

Many encountered problems in science and engineering cannot be modeled by classical partial differential equations. Fortunately, researchers could find a solution for this dilemma. They have discovered a new generation of more general differential equations, the so-called fractional differential equations. These type of equations have been successfully used in modeling many problems in fluid flow and many other different processes and systems such as physical and biological ones. This type of equations appears also in classical mechanics, quantum mechanics, nuclear physics and many other fields. See for example $[4,6-10,16,18,19,30]$. Fractional calculus takes care of the study and applications of integrals as well as time and space derivatives of arbitrary fractional order. If in the ordinary wave equation, we replace the time derivative by the fractional derivative, we obtain the fractional order wave equation. A gold mine of fractional calculus and its essentials can be found in [14, 24, 30, 31]. Most of the fractional order ordinary or partial differential equations have not analytic and exact solutions, therefore many researchers have used numerical methods and techniques to solve time and space fractional initial and boundary value problems and equations. See for example [1, 2, 5, 11-13, 24, 26-28]. Our problem can be placed in the category of fractional order hyperbolic equations, it is in fact a fractional diffusion wave equation with Bessel operator. In the equation (2.1) below, the order $\beta=1+\alpha$ of the time derivative can be any real number in the interval $[0,2]$. The interval $(0,1]$ indicates the diffusion phenomenon, while the interval $(1,2]$ indicates the wave propagation phenomenon. This type of equations can model the propagation of

\footnotetext{
*Corresponding author

Email addresses: mesloub@ksu.edu.sa (Said Mesloub), abachar@ksu.edu.sa (Imed Bachar)
} 
mechanical waves in viscoelastic medium $[17,20,21]$, electromagnetic acoustic, and mechanical responses [29].

We organize the contents of this paper in the following way: In Section 2, we give the setting of the problem and display different types of fractional derivatives needed in this paper. Section 3 is devoted to some preliminaries and some crucial tools needed in the next sections, we also reformulate the posed problem and write it in an operator form which helps establishing some a priori estimates and proving some density results. In Section 4, we prove the uniqueness of the strong solution and its dependence on the given data of the posed problem. This is done by obtaining some a priori bounds based on certain functional differential operator multipliers. In Section 5, we prove the existence of the solution of the problems (2.1), (2.2), (2.3). That is we prove that the closure of the range of the operator L generated by the considered problem is dense in the Hilbert space $Y$ having the norm (3.1) defined below.

\section{Problem setting}

In the rectangle $\mathrm{Q}=(0,1) \times(0, \mathrm{~T}), \mathrm{T}<\infty$, we consider the time fractional initial boundary value problem

$$
\begin{gathered}
c_{\partial_{t}+1} u=\frac{1}{x} \frac{\partial}{\partial x}\left(x \frac{\partial u}{\partial x}\right)-H(x, t) u+f(x, t), \quad x \in(0,1), \quad t \in(0, T), \\
l_{1} u=u(x, 0)=\varphi(x), \quad l_{2} u=u_{t}(x, 0)=\psi(x), \quad x \in(0,1), \\
\lim _{x \rightarrow 0} x u_{x}(x, t)=0, \quad u_{x}(1, t)=0, \quad t \in(0, T),
\end{gathered}
$$

where $H(x, t)$, and $f(x, t)$ are given functions that satisfy certain conditions which will be specified later on.

In our work, we better use Caputo derivative concept rather than Riemann- Liouville derivative, since the initial value of the fractional equation with Caputo derivative is the same as that of integer differential equation.

The operator ${ }^{\mathrm{C}} \partial_{t}^{\alpha+1}$ denotes the Caputo fractional derivative of order $1+\alpha$, with $\alpha \in(0,1)$ defined by

$$
c_{\partial_{t}}^{\alpha+1} u(x, t)=\frac{1}{\Gamma(1-\alpha)} \int_{0}^{t} \frac{u_{\tau \tau}(x, \tau)}{(t-\tau)^{\alpha}} d \tau
$$

where $\Gamma(1-\alpha)$ is the well-known Gamma function.

We assume that there exists a solution belonging to the space $C^{2,2}(\bar{Q})$, the set of functions together with their partial derivatives of order 2 in $x$ and $t$ are continuous on $\bar{Q}$.

The Riemann-Liouville integral of order $0<\alpha<1$ is defined by

$$
D_{t}^{-\alpha} u(x, t)=\frac{1}{\Gamma(\alpha)} \int_{0}^{t} \frac{u(x, \tau)}{(t-\tau)^{1-\alpha}} d \tau
$$

For more and ample information see [10, 30].

Our work can be considered as a further elaboration and generalization of the results obtained in [3], where the author proved the uniqueness of solution of an initial boundary value problem for a fractional wave equation subject to some homogeneous initial and classical boundary conditions. In our case, we have proved the uniqueness as well as the existence of solution for a mixed problem with Bessel operator.

\section{Preliminaries}

The following tools are crucial for proving our results.

Lemma 3.1 ([15]). Let $\mathrm{S}(\mathrm{t})$ be nonnegative and absolutely continuous on $[0, \mathrm{~T}]$, and for almost all $\mathrm{t} \in[0, \mathrm{~T}]$ satisfies the inequality 


$$
\frac{d S}{d t} \leqslant a(t) S(t)+b(t),
$$

where the functions $\mathrm{a}(\mathrm{t})$ and $\mathrm{b}(\mathrm{t})$ are summable and nonnegative on $[0, \mathrm{~T}]$. Then

$$
\begin{aligned}
S(t) & \leqslant e^{\int_{0}^{t} a(\tau) d \tau}\left(S(0)+\int_{0}^{t} b(\xi) \cdot e^{\int_{0}^{\xi} a(\tau) d \tau} d \xi\right) \\
& \leqslant e^{\int_{0}^{t} a(\tau) d \tau}\left(S(0)+\int_{0}^{t} b(\tau) d \tau\right) .
\end{aligned}
$$

Lemma 3.1 can be generalized as follows.

Lemma 3.2 ([3]). Let a nonnegative absolutely continuous function $\mathrm{h}(\mathrm{t})$ satisfy the inequality

$$
\mathrm{c}_{\mathrm{t}^{\alpha}}^{\alpha} \mathrm{h}(\mathrm{t}) \leqslant \mathrm{c}_{1} \mathrm{~h}(\mathrm{t})+\mathrm{c}_{2}(\mathrm{t}), \quad 0<\alpha<1,
$$

for almost all $\mathrm{t} \in[0, \mathrm{~T}]$, where $\mathrm{c}_{1}$ is positive and $\mathrm{c}_{2}(\mathrm{t})$ is an integrable nonnegative function on $[0, \mathrm{~T}]$. Then

$$
h(t) \leqslant h(0) E_{\alpha}\left(c_{1} t^{\alpha}\right)+\Gamma(\alpha) E_{\alpha, \alpha}\left(c_{1} t^{\alpha}\right) D_{t}^{-\alpha} c_{2}(t),
$$

where

$$
E_{\alpha}(x)=\sum_{n=0}^{\infty} \frac{x^{n}}{\Gamma(\alpha n+1)}, \quad \text { and } \quad E_{\alpha, \mu}(x)=\sum_{n=0}^{\infty} \frac{x^{n}}{\Gamma(\alpha n+\mu)},
$$

are the Mittag-Leffler functions.

Lemma 3.3 ([3]). For any absolutely continuous function $w(t)$ on the interval $[0, T]$, the following inequality holds

$$
w(t){ }^{C} \partial_{t}^{\alpha} w(t) \geqslant \frac{1}{2}{ }^{c} \partial_{t}^{\alpha} w^{2}(t), \quad 0<\alpha<1 .
$$

Lemma 3.4 ([23]). If $\mathrm{F}_{\mathrm{k}}(\mathrm{t})(\mathrm{k}=1,2,3)$ are nonnegative functions on $(0, \mathrm{~T}), \mathrm{F}_{1}(\mathrm{t})$ and $\mathrm{F}_{2}(\mathrm{t})$ are integrable functions, and $\mathrm{F}_{3}(\mathrm{t})$ is nondecreasing on $(0, \mathrm{~T})$, then it follows from

$$
\int_{0}^{t} F_{1}(s) d s+F_{2}(t) \leqslant F_{3}(t)+C \int_{0}^{t} F_{2}(s) d s,
$$

that

$$
\int_{0}^{t} F_{1}(s) d s+F_{2}(t) \leqslant e^{C t} F_{3}(t)
$$

The Cauchy $\varepsilon$-inequality is

$$
A B \leqslant \frac{\varepsilon}{2} A^{2}+\frac{1}{2 \varepsilon} B^{2}, \quad \varepsilon>0,
$$

where $A$ and $B$ are positive numbers.

We need the following function spaces: The weighted $\mathrm{L}^{2}$-space, $\mathrm{L}_{\mu}^{2}(\mathrm{Q})$ with inner product

$$
(\mathrm{U}, \mathrm{V})_{\mathrm{L}_{\rho}^{2}(\mathrm{Q})}=(x \mathrm{U}, \mathrm{V})_{\mathrm{L}^{2}(\mathrm{Q})}=\int_{\mathrm{Q}} x \mathrm{UVd} \mathrm{ddt},
$$

and the weighted Sobolev space $W_{2, \mu}^{1,0}(Q)$ with scalar product

$$
(\mathrm{U}, \mathrm{V})_{\mathrm{W}_{2, \mu}^{1,0}(\mathrm{Q})}=(\mathrm{U}, \mathrm{V})_{\mathrm{L}_{\mu}^{2}(\mathrm{Q})}+\left(\mathrm{U}_{\mathrm{x}}, \mathrm{V}_{\mathrm{x}}\right)_{\mathrm{L}_{\mu}^{2}(\mathrm{Q})}
$$

We also use the weighted space on $(0,1)$ such as $L_{\mu}^{2}(0,1)$ and $W_{2, \mu}^{1}(0,1)$ whose definitions are analogous to the spaces on $Q$. 
For establishing the existence and uniqueness of solution of the problems (2.1)-(2.3), we write it in its operator form. This allows us to obtain some energy inequalities needed for our proofs.

The solution of the problems (2.1)-(2.3) can be regarded as the solution of operator equation

$$
\mathrm{Lu}=\mathcal{W}=(\mathrm{f}, \varphi, \psi),
$$

with $L=\left(\mathcal{L}, l_{1}, l_{2}\right)$, and $L: B \rightarrow Y$ is an unbounded operator with domain of definition

$$
D(L)=\left\{\begin{array}{l}
u \in L^{2}(Q), \quad \partial_{t}^{\alpha+1} u, u_{x}, u_{t}, u_{x x} \in L^{2}(Q), \\
\lim _{x \rightarrow 0} x u(x, t)=0, u_{x}(1, t)=0, \quad t \in(0, T) .
\end{array}\right.
$$

where $B$ is a Banach space of the functions $u$ endowed with the finite norm

$$
\|u\|_{B}^{2}=\sup _{0 \leqslant t \leqslant T}\left(D_{t}^{\alpha-1}\left\|u_{t}\right\|_{L_{\mu}^{2}(0,1)}^{2}+\|u\|_{W_{2, \mu}^{1}(0,1)}^{2}\right),
$$

and $Y$ is the Hilbert space constituting of the elements $\mathcal{W}=(f, \varphi, \psi)$ equipped with the norm

$$
\|\mathcal{W}\|_{Y}^{2}=\|f\|_{L_{\mu}^{2}(Q)}^{2}+\|\varphi\|_{W_{2, \mu}^{1}(0,1)}^{2}+\|\psi\|_{L_{\mu}^{2}(0,1)}^{2} .
$$

Here $\mathcal{L}$ is the differential operator

$$
\mathcal{L}={ }^{c} \partial_{t}^{\alpha+1}-\frac{1}{x}\left(x \frac{\partial}{\partial x}\right)_{x}+H
$$

\section{Uniqueness of solution and its dependence on the given data}

Theorem 4.1. Assume that the function $\mathrm{H}(\mathrm{x}, \mathrm{t})$ satisfies the conditions

(i) $\quad c_{3} \leqslant H(x, t) \leqslant c_{4}$,

(ii) $\quad c_{1} \geqslant \frac{\partial H}{\partial t}, \quad c_{2} \geqslant \frac{\partial H}{\partial x}$,

where $c_{j}, j=1,2,3,4$ are positive constants. Then for any $u \in D(L)$, and $f(x, t) \in C(\bar{Q})$, the solution of the problems (2.1)-(2.3) verifies the inequalities

$$
\mathrm{c}_{\partial_{\mathrm{t}}^{\alpha}}^{\alpha}\left\|\mathrm{u}_{\mathrm{t}}\right\|_{\mathrm{L}_{\mu}^{2}(0,1)}^{2}+\frac{\partial}{\partial t} \int_{0}^{1} x \mathrm{u}_{x}^{2} \mathrm{~d} x+\frac{\partial}{\partial t} \int_{0}^{1} x \mathrm{H}(x, t) \mathrm{u}^{2} \mathrm{~d} x \leqslant \delta_{1}\left(\left\|\mathrm{u}_{\mathrm{t}}\right\|_{\mathrm{L}_{\mu}^{2}(0,1)}^{2}+\|\mathrm{u}\|_{\mathrm{W}_{2, \mu}^{1}(0,1)}^{2}+\|f\|_{\mathrm{L}_{\mu}^{2}(0,1)}^{2}\right),
$$

and

$$
\sup _{0 \leqslant t \leqslant T}\left(D_{t}^{\alpha-1}\left\|u_{t}\right\|_{L_{\mu}^{2}(0,1)}^{2}+\|u\|_{W_{2, \mu}^{1}(0,1)}^{2}\right) \leqslant \delta_{6}\left(\int_{0}^{T}\|f\|_{L_{\mu}^{2}(0,1)}^{2} d t+\|\psi\|_{L_{\mu}^{2}(0,1)}^{2}+\|\varphi\|_{W_{2, \mu}^{1}(0,1)}^{2}\right),
$$

for all $\mathrm{u} \in \mathrm{D}(\mathrm{L})$, where

$$
\begin{aligned}
& \delta_{1}=\max \left(c_{1}, 1\right), \quad \delta_{2}=\frac{\delta_{1}}{\min \left(1, c_{3}\right)}, \quad \delta_{3}=\delta_{2} e^{T \delta_{2}} \max \left(T^{2} l 2, T\right), \\
& \delta_{4}=\Gamma(\alpha) E_{\alpha, \alpha}\left(\delta_{3} T^{\alpha}\right) \max \left(\delta_{3}, \frac{\delta_{3} T^{\alpha+1}}{(\alpha+1) \Gamma(\alpha+1)}\right), \\
& \delta_{5}=\max \left(\delta_{2}+\delta_{2} \delta_{3}, \delta_{2} \delta_{3} \delta_{4}+\delta_{2} \delta_{4}\right), \\
& \delta_{6}=\delta_{5}\left(1+\frac{T^{\alpha}}{\Gamma(1+\alpha)}\right) .
\end{aligned}
$$

Proof. For $u \in D(L)$, we consider the identity

$$
\left(c^{c} \partial_{t}^{\alpha+1} u-\frac{1}{x} \frac{\partial}{\partial x}\left(x \frac{\partial u}{\partial x}\right)+H(x, t) u, u_{t}\right)_{L_{\mu}^{2}(0,1)}=\left(f, u_{t}\right)_{L_{\mu}^{2}(0,1)}
$$


Boundary and initial conditions (2.2) and (2.3), yield

$$
\begin{gathered}
\left({ }^{C} \partial_{t}^{\alpha+1} u, u_{t}\right)_{L_{\mu}^{2}(0,1)}=\left({ }^{C} \partial_{t}^{\alpha} u_{t}, u_{t}\right)_{L_{\mu}^{2}(0,1)} \\
-\left(\frac{1}{x} \frac{\partial}{\partial x}\left(x \frac{\partial u}{\partial x}\right), u_{t}\right)_{L_{\mu}^{2}(0,1)}=\frac{1}{2} \frac{\partial}{\partial t} \int_{0}^{1} x u_{x}^{2} d x \\
\left(H(x, t) u, u_{t}\right)_{L_{\mu}^{2}(0,1)}=\frac{1}{2} \frac{\partial}{\partial t} \int_{0}^{1} x H(x, t) u^{2} d x-\frac{1}{2} \int_{0}^{1} x \frac{\partial H}{\partial t} u^{2} d x .
\end{gathered}
$$

Substitution of (4.5), (4.6), (4.7) into (4.4), gives

$$
2\left({ }^{C} \partial_{t}^{\alpha} u_{t}, u_{t}\right)_{L_{\mu}^{2}(0,1)}+\frac{\partial}{\partial t} \int_{0}^{1} x u_{x}^{2} d x+\frac{\partial}{\partial t} \int_{0}^{1} x H(x, t) u^{2} d x=\int_{0}^{1} x \frac{\partial H}{\partial t} u^{2} d x+2\left(f, u_{t}\right)_{L_{\mu}^{2}(0,1)} .
$$

Thanks to Lemma 3.3 in [3] which allows us to estimate the first term on the LHS of (4.8) as follows

$$
2\left({ }^{c} \partial_{t}^{\alpha} u_{t}, u_{t}\right)_{L_{\mu}^{2}(0,1)} \geqslant{ }^{c} \partial_{t}^{\alpha}\left\|u_{t}\right\|_{L_{\mu}^{2}(0,1)}^{2} .
$$

Conditions (ii) in (4.1), Cauchy $\varepsilon$-inequality and combination of inequality (4.9) and equality (4.8), yield

$$
\mathrm{C}_{\partial_{\mathrm{t}}^{\alpha}}^{\alpha}\left\|\mathrm{u}_{\mathrm{t}}\right\|_{\mathrm{L}_{\mu}^{2}(0,1)}^{2}+\frac{\partial}{\partial t} \int_{0}^{1} x \mathrm{u}_{x}^{2} \mathrm{~d} x+\frac{\partial}{\partial t} \int_{0}^{1} x \mathrm{H}(x, t) \mathrm{u}^{2} \mathrm{~d} x \leqslant \delta_{1}\left(\|f\|_{\mathrm{L}_{\mu}^{2}(0,1)}^{2}+\left\|\mathrm{u}_{\mathrm{t}}\right\|_{\mathrm{L}_{\mu}^{2}(0,1)}^{2}+\|\mathrm{u}\|_{W_{2, \mu}^{1}(0,1)}^{2}\right),
$$

where

$$
\delta_{1}=\max \left(c_{1}, 1\right)
$$

This achieves the proof of estimate (4.2).

By replacing $t$ by $\tau$ and integrating both sides of (4.10) with respect to $\tau$ from 0 to $t$ and using condition (i) in (4.1), we obtain

$$
\begin{aligned}
D_{t}^{\alpha-1}\left\|u_{\mathfrak{t}}\right\|_{L_{\mu}^{2}(0,1)}^{2}+\|u\|_{W_{2, \mu}^{1}(0,1)}^{2} \leqslant & \delta_{2}\left(\int_{0}^{t}\left\|u_{\tau}(x, \tau)\right\|_{L_{\mu}^{2}(0,1)}^{2} d \tau+\int_{0}^{t}\|u(x, \tau)\|_{W_{2, \mu}^{1}(0,1)}^{2} d \tau+\int_{0}^{t}\|f\|_{L_{\mu}^{2}(0,1)}^{2} d \tau\right. \\
& \left.+\|\psi\|_{L_{\mu}^{2}(0,1)}^{2}+\|\varphi\|_{W_{2, \mu}^{1}(0,1)}^{2}\right)
\end{aligned}
$$

where

$$
\delta_{2}=\frac{\max \left(\delta_{1}, 1, c_{4}, T^{1-\alpha}\right)}{\min \left(1, c_{3}\right)}
$$

If we discard the first term on the LHS in (4.11) and apply Lemma 3.1 in [15] by taking

$$
S(t)=\int_{0}^{t}\|u(x, \tau)\|_{W_{\mu}^{1,2}(0,1)}^{2} d \tau,
$$

and

$$
S^{\prime}(t)=\|u\|_{W_{2, \mu}^{1}(0,1)}^{2}, \quad S(0)=0,
$$

we obtain

$$
\begin{aligned}
\int_{0}^{t}\|\mathfrak{u}(x, \tau)\|_{W_{2, \mu}^{1}(0,1)}^{2} \mathrm{~d} \tau \leqslant & \delta_{3}\left(\int_{0}^{t}\left\|u_{\tau}(x, \tau)\right\|_{\mathrm{L}_{\mu}^{2}(0,1)}^{2} \mathrm{~d} \tau\right. \\
& \left.+\int_{0}^{t}\|f\|_{\mathrm{L}_{\mu}^{2}(0,1)}^{2} \mathrm{~d} \tau+\|\psi\|_{\mathrm{L}_{\mu}^{2}(0,1)}^{2}+\|\varphi\|_{W_{2, \mu}^{1}(0,1)}^{2}\right),
\end{aligned}
$$


where

$$
\delta_{3}=\delta_{2} e^{T \delta_{2}} \max \left(T^{2} l 2, T\right) .
$$

Now by discarding the last term on the LHS of (4.11) and using (4.12), we get

$$
\begin{aligned}
D_{t}^{\alpha-1}\left\|u_{t}\right\|_{L_{\mu}^{2}(0,1)}^{2} \leqslant & \delta_{3}\left(\int_{0}^{t}\left\|u_{\tau}(x, \tau)\right\|_{L_{\mu}^{2}(0,1)}^{2} d \tau\right. \\
& \left.+\int_{0}^{t}\|f\|_{L_{\mu}^{2}(0,1)}^{2} d \tau+\|\psi\|_{L_{\mu}^{2}(0,1)}^{2}+\|\varphi\|_{W_{2, \mu}^{1}(0,1)}^{2}\right) .
\end{aligned}
$$

Lemma 3.2 can now be applied, where we have

$$
\begin{aligned}
h(t) & =\int_{0}^{t}\left\|u_{\tau}(x, \tau)\right\|_{L_{\mu}^{2}(0,1)}^{2} d \tau, \quad h(0)=0, \\
\partial_{t}^{\alpha} h(t) & =D_{t}^{\alpha-1}\left\|u_{t}\right\|_{L_{\mu}^{2}(0,1)}^{2} .
\end{aligned}
$$

In this regard, we have the estimate

$$
\int_{0}^{t}\left\|u_{\tau}(x, \tau)\right\|_{L_{\mu}^{2}(0,1)}^{2} d \tau \leqslant \delta_{4}\left(D_{t}^{-\alpha-1}\|f\|_{L_{\mu}^{2}(0,1)}^{2}+\|\psi\|_{L_{\mu}^{2}(0,1)}^{2}+\|\varphi\|_{W_{2, \mu}^{1}(0,1)}^{2}\right),
$$

where

$$
\delta_{4}=\Gamma(\alpha) \mathrm{E}_{\alpha, \alpha}\left(\delta_{3} T^{\alpha}\right) \max \left(\delta_{3}, \frac{\delta_{3} T^{\alpha+1}}{(\alpha+1) \Gamma(\alpha+1)}\right) .
$$

Combination of inequalities (4.11), (4.12), and (4.13) gives

$$
\begin{aligned}
D_{t}^{\alpha-1}\left\|u_{t}\right\|_{L_{\mu}^{2}(0,1)}^{2}+\|u\|_{W_{2, \mu}^{1}(0,1)}^{2} \leqslant & \delta_{5}\left(D_{t}^{-\alpha-1}\|f\|_{L_{\mu}^{2}(0,1)}^{2}+\int_{0}^{t}\|f\|_{L_{\mu}^{2}(0,1)}^{2} d \tau\right. \\
& \left.+\|\psi\|_{L_{\mu}^{2}(0,1)}^{2}+\|\varphi\|_{W_{2, \mu}^{1}(0,1)}^{2}\right)
\end{aligned}
$$

where

$$
\delta_{5}=\max \left(\delta_{2}+\delta_{2} \delta_{3}, \delta_{2} \delta_{3} \delta_{4}+\delta_{2} \delta_{4}\right)
$$

The inequality

$$
D_{t}^{-\alpha-1}\|f\|_{L_{\mu}^{2}(0,1)}^{2} \leqslant \frac{T^{\alpha}}{\Gamma(1+\alpha)} \int_{0}^{t}\|f\|_{L_{\mu}^{2}(0,1)}^{2} d \tau,
$$

reduces $(4.14)$ to

$$
\begin{aligned}
D_{t}^{\alpha-1}\left\|u_{t}\right\|_{L_{\mu}^{2}(0,1)}^{2}+\|u\|_{W_{2, \mu}^{1}(0,1)}^{2} & \leqslant \delta_{6}\left(\int_{0}^{t}\|f\|_{L_{\mu}^{2}(0,1)}^{2} d \tau+\|\psi\|_{L_{\mu}^{2}(0,1)}^{2}+\|\varphi\|_{W_{2, \mu}^{1}(0,1)}^{2}\right) \\
& \leqslant \delta_{6}\left(\int_{0}^{T}\|f\|_{L_{\mu}^{2}(0,1)}^{2} d \tau+\|\psi\|_{L_{\mu}^{2}(0,1)}^{2}+\|\varphi\|_{W_{2, \mu}^{1}(0,1)}^{2}\right)
\end{aligned}
$$

where

$$
\delta_{6}=\delta_{5}\left(1+\frac{T^{\alpha}}{\Gamma(1+\alpha)}\right) .
$$

Since the right-hand side of (4.15) does not depend on $t$, we can replace the left-hand side by its upper bound with respect to $t$ over $(0, T)$ which finishes the proof of estimate (4.3) from which we deduce the uniqueness and continuous dependence of the solution of the problems (2.1)-(2.3) on the given data. 
Before proceeding to the proof of the existence of the solution of the problems (2.1)-(2.3), we mention some consequences of the estimate (4.3).

We can deduce from the a priori estimate (4.3) that there exists an inverse non bounded operator $L^{-1}: R(L) \rightarrow B$. Since the range of $L$ is a subset of $Y$, we construct its closure $\bar{L}$ so that the estimate (4.3) holds for the extension and that the range of $\bar{L}$ coincides with the whole space $Y$. In this regard, we can show that $\mathrm{L}: \mathrm{B} \rightarrow \mathrm{Y}$ admits a closure as stated in the following.

Proposition 4.2. The operator $\mathrm{L}: \mathrm{B} \rightarrow \mathrm{Y}$ has a closure $\overline{\mathrm{L}}$.

Proof. The proof is similar to that in [22].

We then define the strong solution of the problems (2.1)-(2.3) as the solution of the operator equation:

$$
\overline{\mathrm{L}} \mathrm{u}=\mathcal{W}=(f, \varphi, \psi),
$$

and the estimate (4.3) can be extended to

$$
\|\mathrm{u}\|_{\mathrm{B}} \leqslant \sqrt{\delta_{6}}\|\overline{\mathrm{L}} \mathrm{u}\|_{Y}, \quad \forall \mathrm{u} \in \mathrm{D}(\overline{\mathrm{L}}) .
$$

From this last inequality (4.16), we deduce the uniqueness of the strong solution and that:

Corollary 4.3. The range of $\overline{\mathrm{L}}$ is closed in $\mathrm{Y}$ and the closure of the range of $\mathrm{L}$ coincides with the range of the closure of $\overline{\mathrm{L}}$, and $\overline{\mathrm{L}}^{-1}=\overline{\mathrm{L}^{-1}}$, where $\overline{\mathrm{L}^{-1}}$ is the extension by continuity from the range of $\mathrm{L}$ to the closure of its range.

\section{Solvability of the posed problem}

To prove the existence of solution of our problems (2.1)-(2.3), we use a density argument. In fact, we demonstrate that the range $R(L)$ of the operator $L$ is dense in $Y$ for every element $w$ in the Banach space B.

We now state the results of the existence of solution of our fractional problems (2.1)-(2.3).

Theorem 5.1. Assume that conditions of Theorem 4.1 are satisfied. Then for all $\mathcal{W}=(f, \varphi, \psi) \in Y$, there exists a unique strong solution $\mathrm{u}=\overline{\mathrm{L}}^{-1} \mathcal{W}=\overline{\mathrm{L}^{-1}} \mathcal{W}$ of the problems (2.1)-(2.3).

Proof. Estimate (4.16) asserts that, if a strong solution of (2.1)-(2.3) exists, it is unique and depends continuously on the data. Corollary 4.3 says that in order to prove that problem (2.1)-(2.3) admits a strong solution for any $\mathcal{W}=(f, \varphi, \psi) \in Y$, it suffices to show that the closure of the range of $L$ is dense in $Y$. We first prove the following special case of density

Proposition 5.2. If for some function $\mathrm{G}(\mathrm{x}, \mathrm{t}) \in \mathrm{L}_{\mu}^{2}(\mathrm{Q})$, and all $\mathrm{K}(\mathrm{x}, \mathrm{t}) \in \mathrm{D}(\mathrm{L})$ satisfying homogeneous initial conditions $l_{1} \mathrm{~K}=0, l_{2} \mathrm{~K}=0$, we have

$$
(\mathcal{L K}, \mathrm{G})_{\mathrm{L}_{\mu}^{2}(\mathrm{Q})}=0 \text {, }
$$

then $\mathrm{G}$ is zero a.e in the domain $\mathrm{Q}$.

Proof. Equation (5.1) implies

$$
\left({ }^{C} \partial_{t}^{\alpha+1} K-\frac{1}{x} \frac{\partial}{\partial x}\left(x \frac{\partial K}{\partial x}\right)+H(x, t) K, G\right)_{L_{\mu}^{2}(Q)}=0 .
$$

Let $p(x, t)$ be a function satisfying conditions (2.2) and (2.3) and such that

$$
p, p_{x}, \int_{0}^{t} p(x, s) d s, x \int_{0}^{t} \frac{\partial p(x, s)}{\partial x} d s, \partial_{t}^{\alpha+1} p \in L^{2}(Q),
$$


we then set

$$
K(x, t)=\int_{0}^{t} \int_{0}^{s} p(x, z) d z d s
$$

Equation (5.2) then becomes

$$
\left.\left(C_{\partial_{t}^{\alpha+1}}^{\alpha+x} \int_{0}^{t} \int_{0}^{s} p(x, z) d z d s\right)-\frac{\partial}{\partial x}\left(x \int_{0}^{t} \int_{0}^{s} p_{x}(x, z) d z d s\right)+x H(x, t)\left(\int_{0}^{t} \int_{0}^{s} p(x, z) d z d s\right), G\right)_{L^{2}(Q)}=0 .
$$

Let us now consider the function

$$
\mathrm{G}(x, \mathrm{t})=x \int_{0}^{t} p(x, s) d s
$$

It is quite obvious that the function $G(x, t)$ belongs to $L^{2}(Q)$.

Equations (5.3) and (5.4) lead to

$$
\begin{aligned}
\left(\mathrm{c}_{\partial_{\mathrm{t}}^{\alpha+1}}\right. & \left.\left(\int_{0}^{\mathrm{t}} \int_{0}^{s} \mathrm{p}(x, z) \mathrm{d} z \mathrm{~d} s\right), \int_{0}^{\mathrm{t}} \mathrm{p}(x, \mathrm{~s}) \mathrm{ds}\right)_{\mathrm{L}_{\mu}^{2}(\mathrm{Q})} \\
& -\left(\frac{\partial}{\partial x}\left(x \int_{0}^{\mathrm{t}} \int_{0}^{s} p_{x}(x, z) \mathrm{d} z \mathrm{~d} s\right), \int_{0}^{\mathrm{t}} \mathrm{p}(x, s) \mathrm{d} s\right)_{\mathrm{L}^{2}(\mathrm{Q})} \\
& +\left(x \mathrm{H}(x, \mathrm{t}) \int_{0}^{\mathrm{t}} \int_{0}^{s} \mathrm{p}(x, z) \mathrm{d} z \mathrm{~d} s, \int_{0}^{\mathrm{t}} \mathrm{p}(x, s) \mathrm{d} s\right)_{\mathrm{L}_{\mu}^{2}(\mathrm{Q})}=0 .
\end{aligned}
$$

Put in mind that the function $p$ verifies the boundary and initial conditions (2.2) and (2.3), then we have

$$
\begin{aligned}
\left(c_{\partial_{t}}^{\alpha+1}\left(\int_{0}^{t} \int_{0}^{s} p(x, z) d z d s\right), \int_{0}^{t} p(x, s) d s\right)_{L_{\mu}^{2}(0,1)} & =\left(c_{t} \partial_{t}^{\alpha}\left(\int_{0}^{t} p(x, s) d s\right), \int_{0}^{t} p(x, s) d s\right)_{L_{\mu}^{2}(0,1)} \\
-\left(\frac{\partial}{\partial x}\left(x \int_{0}^{t} \int_{0}^{s} p_{x}(x, z) d z d s\right), \int_{0}^{t} p(x, s) d s\right)_{L^{2}(0,1)} & =\left(\int_{0}^{t} p_{x}(x, s) d s, \int_{0}^{t} \int_{0}^{s} p_{x}(x, z) d z d s\right)_{L_{\mu}^{2}(0,1)} \\
& =\frac{1}{2} \frac{\partial}{\partial t} \int_{0}^{1} x\left(\int_{0}^{t} \int_{0}^{s} p_{x}(x, z) d z d s\right)^{2} d x, \\
\left(x H(x, t) \int_{0}^{t} \int_{0}^{s} p(x, z) d z d s, \int_{0}^{t} p(x, s) d s\right)_{L_{\mu}^{2}(0,1)}= & \frac{1}{2} \frac{\partial}{\partial t} \int_{0}^{1} x^{2} H(x, t)\left(\int_{0}^{t} \int_{0}^{s} p(x, z) d z d s\right)^{2} d x \\
& -\frac{1}{2} \int_{0}^{1} x^{2} \frac{\partial H}{\partial t}\left(\int_{0}^{t} \int_{0}^{s} p(x, z) d z d s\right)^{2} d x .
\end{aligned}
$$

Insertion of equations (5.6), (5.7), (5.8) into (5.5), yields

$$
\begin{gathered}
2\left({ }_{\partial_{t} \alpha}\left(\int_{0}^{t} p(x, s) d s\right), \int_{0}^{t} p(x, s) d s\right)_{L_{\mu}^{2}(0,1)} \\
+\frac{\partial}{\partial t} \int_{0}^{1} x\left(\int_{0}^{t} \int_{0}^{s} p_{x}(x, z) d z d s\right)^{2} d x \\
+\frac{\partial}{\partial t} \int_{0}^{1} x^{2} H(x, t)\left(\int_{0}^{t} \int_{0}^{s} p(x, z) d z d s\right)^{2} d x \\
\quad=\int_{0}^{1} x^{2} \frac{\partial H}{\partial t}\left(\int_{0}^{t} \int_{0}^{s} p(x, z) d z d s\right)^{2} d x .
\end{gathered}
$$


Lemma 3.3 allows us to estimate the first term on the LHS of (5.9) as follows

$$
2\left(c^{c} \partial_{t}^{\alpha}\left(\int_{0}^{t} p(x, s) d s\right), \int_{0}^{t} p(x, s) d s\right)_{L_{\mu}^{2}(0,1)} \geqslant c^{c} \partial_{t}^{\alpha}\left\|\int_{0}^{t} p(x, s) d s\right\|_{L_{\mu}^{2}(0,1)}^{2} .
$$

Consequently, in light of condition (ii) in (4.1) and (5.10), equality (5.9) becomes

$$
\begin{aligned}
& c_{\partial_{t}^{\alpha}}^{\alpha}\left\|\int_{0}^{t} p(x, s) d s\right\|_{L_{\mu}^{2}(0,1)}^{2}+\frac{\partial}{\partial t} \int_{0}^{1} x\left(\int_{0}^{t} \int_{0}^{s} p_{x}(x, z) d z d s\right)^{2} d x+\frac{\partial}{\partial t} \int_{0}^{1} x^{2} H(x, t)\left(\int_{0}^{t} \int_{0}^{s} p(x, z) d z d s\right)^{2} d x \\
& \leqslant c_{1} \int_{0}^{1} x^{2}\left(\int_{0}^{t} \int_{0}^{s} p(x, z) d z d s\right)^{2} d x
\end{aligned}
$$

By replacing $t$ by $\tau$ in (5.11) and then integrating with respect to $\tau$ from 0 to $t$, we obtain

$$
\begin{aligned}
& D_{\mathrm{t}}^{\alpha-1}\left\|\int_{0}^{\mathrm{t}} \mathrm{p}(x, \mathrm{~s}) \mathrm{ds}\right\|_{\mathrm{L}_{\mu}^{2}(0,1)}^{2}+\int_{0}^{1} x\left(\int_{0}^{\mathrm{t}} \int_{0}^{s} p_{x}(x, z) \mathrm{d} z \mathrm{~d} s\right)^{2} \mathrm{~d} x+\int_{0}^{1} x^{2} \mathrm{H}(x, \mathrm{t})\left(\int_{0}^{\mathrm{t}} \int_{0}^{s} p(x, z) \mathrm{d} z \mathrm{~d} s\right)^{2} \mathrm{~d} x \\
& \quad \leqslant c_{1} \int_{0}^{\mathrm{t}} \int_{0}^{1} x^{2}\left(\int_{0}^{\tau} \int_{0}^{s} p(x, z) \mathrm{d} z \mathrm{ds}\right)^{2} \mathrm{~d} x \mathrm{~d} \tau .
\end{aligned}
$$

Condition (i) in (4.1) reduces (5.12) to

$$
\begin{aligned}
& D_{\mathrm{t}}^{\alpha-1}\left\|\int_{0}^{\mathrm{t}} \mathrm{p}(x, \mathrm{~s}) \mathrm{ds}\right\|_{\mathrm{L}_{\mu}^{2}(0,1)}^{2}+\int_{0}^{1} x\left(\int_{0}^{\mathrm{t}} \int_{0}^{s} p_{x}(x, z) \mathrm{d} z \mathrm{ds}\right)^{2} \mathrm{~d} x+\int_{0}^{1} x^{2}\left(\int_{0}^{\mathrm{t}} \int_{0}^{s} p(x, z) \mathrm{d} z \mathrm{ds}\right)^{2} \mathrm{~d} x \\
& \leqslant \frac{\mathrm{c}_{1}}{\min \left(\mathrm{c}_{3}, 1\right)} \int_{0}^{\mathrm{t}} \int_{0}^{1} x^{2}\left(\int_{0}^{\tau} \int_{0}^{s} p(x, z) \mathrm{d} z \mathrm{ds}\right)^{2} \mathrm{~d} x \mathrm{~d} \tau .
\end{aligned}
$$

Application of Lemma 3.4 in [23] to inequality (5.13), yields

$$
\begin{gathered}
D_{t}^{\alpha-1}\left\|\int_{0}^{t} p(x, s) d s\right\|_{L_{\mu}^{2}(0,1)}^{2}+\int_{0}^{1} x\left(\int_{0}^{t} \int_{0}^{s} p_{x}(x, z) d z d s\right)^{2} d x \\
+\int_{0}^{t} \int_{0}^{1} x^{2}\left(\int_{0}^{\tau} \int_{0}^{s} p(x, z) d z d s\right)^{2} d x d \tau \leqslant 0 .
\end{gathered}
$$

Consequently, inequality (5.14) implies that $\mathrm{G}$ is zero a.e in $\mathrm{Q}$.

To complete the proof of Theorem 5.1, we assume that for $\left(\Psi, \omega_{1}, \omega_{2}\right) \in R(L)^{\perp}$, we have

$$
(\mathcal{L} u, \Psi)_{\mathrm{L}_{\mu}^{2}(\mathrm{Q})}+\left(\mathrm{l}_{1} u, w_{1}\right)_{\mathrm{W}_{2, \mu}^{1}(0,1)}+\left(\mathrm{l}_{2} \mathrm{u}, \mathrm{w}_{2}\right)_{\mathrm{L}_{\mu}^{2}(0,1)}=0,
$$

then we should show that $\Psi=0, \omega_{1}=0, \omega_{2}=0$. If we put $u \in D(L)$ satisfying conditions $u(x, 0)=0$, $u_{\mathfrak{t}}(x, 0)=0$ into (5.15), we get

$$
(\mathcal{L u}, \Psi)_{\mathrm{L}_{\mu}^{2}(\mathrm{Q})}=0, \quad \forall \mathrm{u} \in \mathrm{D}(\mathrm{L}) .
$$

By applying Proposition 4.2 to (5.16), we see that $\Psi=0$. Consequently (5.15) becomes

$$
\left(l_{1} u, \omega_{1}\right)_{W_{2, \mu}^{1}(0,1)}+\left(l_{2} u, \omega_{2}\right)_{L_{\mu}^{2}(0,1)}=0, \quad \forall u \in D(L) .
$$

The fact that the ranges of the trace operators $l_{1}$ and $l_{2}$ are respectively dense in the spaces $W_{2, \mu}^{1}(0,1)$ and $\mathrm{L}_{\mu}^{2}(0,1)$, we conclude from (5.17) that $\omega_{1}=0, \omega_{2}=0$, and Theorem 5.1 then follows. 


\section{Acknowledgment}

The authors would like to extend their sincere appreciation to the Deanship of Scientific Research at King Saud University for its funding this Research group No (RG-1435-043). They also would like to thank the referee for his fruitful comments and suggestions.

\section{References}

[1] G. Adomian, A review of the decomposition method in applied mathematics, J. Math. Anal. Appl., 135 (1988), 501-544. 1

[2] G. Adomian, Solving frontier problems of physics: the decomposition method, With a preface by Yves Cherruault, Fundamental Theories of Physics, Kluwer Academic Publishers Group, Dordrecht, (1994). 1

[3] A. A. Alikhanov, A priori estimates for solutions of boundary value problems for fractional-order equations, Dier. Equ., 46 (2010), 660-666. 2, 3.2, 3.3, 4

[4] L. Debnath, D. D. Bhatta, Solutions to few linear fractional inhomogeneous partial differential equations in fluid mechanics, Fract. Calc. Appl. Anal., 7 (2004), 21-36. 1

[5] A. M. A. El-Sayed, M. Gaber, The Adomian decomposition method for solving partial differential equations of fractal order in finite domains, Phys. Lett. A, 359 (2006), 175-182. 1

[6] N. Engheta, On fractional calculus and fractional multipoles in electromagnetism, IEEE Trans. Antennas and Propagation, 44 (1996), 554-566. 1

[7] A. Freed, K. Diethelm, Y. Luchko, Fractional-order viscoelasticity (FOV): Constitutive development using the fractional calculus: First annual Report, NASA Technical Reports Server (NTRS), United States, (2002).

[8] R. Gorenfo, Abel integral equations with special emphasis on applications, Lectures in Mathematical Sciences, The University of Tokyo, Graduate School of Mathematical Sciences, (1996).

[9] J. H. He, Some applications of nonlinear fractional differential equations and their approximations, Bull. Sci. Technol., 15 (1999), 86-90.

[10] R. Hilfer (Ed.), Applications of fractional calculus in physics, World Scientific Publishing Co., Inc., River Edge, NJ, (2000). 1, 2

[11] H. Jafari, V. Daftardar-Gejji, Solving linear and nonlinear fractional diffusion and wave equations by Adomian decomposition, Appl. Math. Comput., 180 (2006), 488-497. 1

[12] T. Kaplan, L. J. Gray, S. H. Liu, Self-affine fractal model for a metal-electrolyte interface, Phys. Rev. B, 35 (1987), 5379 5381.

[13] A. M. Keighttey, J. C. Myland, K. B. Oldham, P. G. Symons, Reversible cyclic voltammetry in the presence of product, J. Electroanal. Chem., 322 (1992), 25-54. 1

[14] A. A. Kilbas, H. M. Srivastava, J. J. Trujillo, Theory and applications of fractional differential equations, North-Holland Mathematics Studies, Elsevier Science B.V., Amsterdam, (2006). 1

[15] O. A. Ladyzhenskaya, The boundary value problems of mathematical physics, Translated from the Russian by Jack Lohwater [Arthur J. Lohwater], Applied Mathematical Sciences, Springer-Verlag, New York, (1985). 3.1, 4

[16] A. Le Mehaute, G. Crepy, Introduction to transfer and motion in fractal media: the geometry of kinetics, Solid State Ion., 9 (1983), 17-30. 1

[17] F. Mainardi, Fractional relaxation-oscillation and fractional diffusion-wave phenomena, Chaos Solitons Fractals, 7 (1996), 1461-1477. 1

[18] F. Mainardi, Fractional calculus: some basic problems in continuum and statistical mechanics, Fractals and fractional calculus in continuum mechanics, Udine, (1996), 291-348, CISM Courses and Lectures, Springer, Vienna, (1997). 1

[19] F. Mainardi, Fractional calculus and waves in linear viscoelasticity, An introduction to mathematical models. Imperial College Press, London, (2010). 1

[20] F. Mainardi, Y. Luchko, G. Pagnini, The fundamental solution of the space-time fractional diffusion equation, Fract. Calc. Appl. Anal., 4 (2001), 153-192. 1

[21] F. Mainardi, P. Paradisi, Fractional diffusive waves, J. Comput. Acoust., 9 (2001), 1417-1436. 1

[22] S. Mesloub, A. Bouziani, On a class of singular hyperbolic equation with a weighted integral condition, Int. J. Math. Math. Sci., 22 (1999), 511-519. 4

[23] S. Mesloub, R. Mezhoudi, M. Medjeden, A mixed problem for a parabolic equation of higher order with integral conditions, Bull. Polish Acad. Sci. Math., 50 (2012), 313-322. 3.4, 5

[24] K. S. Miller, B. Ross, An introduction to the fractional calculus and fractional differential equations, A Wiley-Interscience Publication. John Wiley \& Sons, Inc., New York, (1993). 1

[25] S. Momani, An explicit and numerical solutions of the fractional KdV equation, Math. Comput. Simulation, 70 (2005), 110-118.

[26] S. Momani, Non-perturbative analytical solutions of the space- and time-fractional Burgers equations, Chaos Solitons Fractals, 28 (2006), 930-937. 1 
[27] S. Momani, R. Qaralleh, Numerical approximations and Padé approximants for a fractional population growth model, Appl. Math. Model., 31 (2007), 1907-1914.

[28] S. Momani, N. Shawagfeh, Decomposition method for solving fractional Riccati differential equations, Appl. Math. Comput., 182 (2006), 1083-1092. 1

[29] R. Nigmatullin, The realization of the generalized transfer equation in a medium with fractal geometry, Phys. Status Solidi B, 133 (1986), 425-430. 1

[30] I. Podlubny, Fractional differential equations, An introduction to fractional derivatives, fractional differential equations, to methods of their solution and some of their applications, Mathematics in Science and Engineering, Academic Press, Inc., San Diego, CA, (1999). 1, 2

[31] S. G. Samko, A. A. Kilbas, O. I. Marichev, Fractional integrals and derivatives, Theory and applications, Edited and with a foreword by S. M. Nikolskiü, Translated from the 1987 Russian original, Revised by the authors, Gordon and Breach Science Publishers, Yverdon, (1993). 1 